\title{
ON SOME ANALYTICAL PROPERTIES OF THE BARNES G-FUNCTION
}

\author{
SUNDAY SANDOW, KWARA NANTOMAH*, BABA SEIDU \\ Department of Mathematics, Faculty of Mathematical Sciences, \\ C. K. Tedam University of Technology and Applied Sciences, \\ P. O. Box 24, Navrongo, UE/R, Ghana \\ *Corresponding author: knantomah@cktutas.edu.gh
}

Received Nov. 5, 2020

\begin{abstract}
We establish an explicit representation for the logarithmic derivative of the Barnes $G$-function which we denote by $\Psi(x)$. We achieve this by using an identity of the classical digamma function, the infinite product form of the Barnes $G$-function and the functional equation of the Barnes $G$-function. We further establish some analytical properties such as monotonicity, complete monotonicity, convexity, log-convexity, subadditivity, superadditivity and starshapedness of the function $\Psi(x)$. Subsequently, we deduce some inequalities involving the Barnes $G$-function.
\end{abstract}

2010 Mathematics Subject Classification: 33B15, 26A48.

Key words and phrases: Barnes G-function; double gamma function; gamma function; digamma function; inequality.

\section{INTRODUCTION}

The classical Euler's gamma function is usually defined for $x>0$ as

$$
\Gamma(x)=\int_{0}^{\infty} t^{x-1} e^{-t} d t
$$

and satisfies the basic properties

$$
\begin{aligned}
& \Gamma(x+1)=x \Gamma(x), \quad x \in \mathbb{R}, \\
& \Gamma(n+1)=n !, \quad n \in \mathbb{N} .
\end{aligned}
$$

DOI: $10.28924 / \mathrm{APJM} / 8-7$ 
Undoubtedly, it is one of the most important special functions. Its usefulness in mathematics as well as other related disciplines is invaluable. The logarithmic derivative of the gamma function which is called the digamma (or psi) function is defined as

$$
\psi(x)=\frac{d}{d x} \ln \Gamma(x)=\frac{\Gamma^{\prime}(x)}{\Gamma(x)}, \quad x>0,
$$

and satisfies the following identities ( [1], [3], [15]).

$$
\begin{aligned}
\psi(x) & =-\gamma-\frac{1}{x}+\sum_{k=1}^{\infty} \frac{x}{k(k+x)} \\
& =-\gamma+\sum_{k=0}^{\infty}\left(\frac{1}{k+1}-\frac{1}{k+x}\right), \\
& =-\gamma+\int_{0}^{\infty} \frac{e^{-t}-e^{-x t}}{1-e^{-t}} d t \\
& =\int_{0}^{\infty}\left(\frac{e^{-t}}{t}-\frac{e^{-x t}}{1-e^{-t}}\right) d t
\end{aligned}
$$

where $\gamma=0.57721 \ldots$ is the Euler-Mascheroni's constant. It also satisfies the functional equation $([1],[3])$

$$
\begin{aligned}
\psi(x+1) & =\frac{1}{x}+\psi(x), \\
& =-\gamma+\sum_{k=1}^{\infty}\left(\frac{1}{k}-\frac{1}{k+x}\right) .
\end{aligned}
$$

The $n$-th derivative of the digamma function which is called the polygamma function of order $n$ satisfies the properties ( [1], [3])

$$
\begin{aligned}
\psi^{(n)}(x) & =\frac{d^{n}}{d x^{n}} \psi(x)=\frac{d^{n+1}}{d x^{n+1}} \ln \Gamma(x) \\
& =(-1)^{n+1} \int_{0}^{\infty} \frac{t^{n} e^{-x t}}{1-e^{-t}} d t \\
& =(-1)^{n+1} n ! \sum_{k=0}^{\infty} \frac{1}{(k+x)^{n+1}},
\end{aligned}
$$

where $x>0$ and $n \in \mathbb{N}$. 
The multiple gamma function $\Gamma_{n}(x)$ which is a generalization of the classical gamma function was introduced by Barnes [5] and is defined by the following recurrence functional equation.

$$
\begin{aligned}
\Gamma_{n}(x+1) & =\frac{\Gamma_{n+1}(x)}{\Gamma_{n}(x)}, \quad x>0, n \in \mathbb{N}, \\
\Gamma_{1}(x) & =\Gamma(x), \\
\Gamma_{n}(1) & =1 .
\end{aligned}
$$

The Barnes' G-function (also known in some text as the double gamma function) is defined as [2]

$$
G(x)=\frac{1}{\Gamma_{2}(x)}, \quad x>0,
$$

and satisfies the properties [14]

$$
\begin{aligned}
G(x+1) & =\Gamma(x) G(x), \\
G(1) & =1, \\
G(m) & =1 ! 2 ! \ldots(m-3) !(m-2) !, \quad m \geq 2 .
\end{aligned}
$$

The infinite product form of the function is given as [4]

$$
G(x+1)=(2 \pi)^{\frac{x}{2}} \exp \left(-\frac{x+x^{2}(1+\gamma)}{2}\right) \prod_{k=1}^{\infty}\left(1+\frac{x}{k}\right)^{k} \exp \left(\frac{x^{2}}{2 k}-x\right) .
$$

Since its introduction, the Barnes $G$-function has caught the attention of several researchers and has been studied in diverse ways. For example, Choi [10] established a duplication formula for the double gamma function $\Gamma_{2}$. Afterwards, Choi and Srivastava [11] obtained a multiplication formula for the function $\Gamma_{n}$. Then Batir and Cancan [7] established upper and lower bounds for the $G$-function in terms of polygamma functions. A year later, Batir [6] further established new lower and upper bounds for the $G$-function in terms of the gamma and digamma functions. Chen [9] extended the results of [6] by obtaining a class of double inequalities for the $G$-function. Also, Adamchik [2] gave a family of integral representations and asymptotic series of the multiple gamma function. Das and Swaminathan [13] also established bounds for ratios of double and tripple gamma functions. Then rencently, $\mathrm{Xu}$ and Wang [19] established two general asymptotic expansions for the $G$-function.

In the present study, we focus our attention on the logarithmic derivative of the Barnes $G$-function which we shall denote by $\Psi(x)$. Our goal is to derive an explicit expression for 
$\Psi(x)$ and to further establish some analytical properties of it. Subsequently, we deduced some inequalities for the Barnes $G$-function.

\section{Auxiliary Definitions and Lemmas}

Definition 2.1. A function $h: I \rightarrow \mathbb{R}$ is said to be convex on I if

$$
h^{\prime \prime}(x) \geq 0,
$$

for all $x \in I$ or equivalently, if

$$
h\left(\frac{x}{r}+\frac{y}{s}\right) \leq \frac{h(x)}{r}+\frac{h(y)}{s},
$$

for all $x, y \in I$, where $r>1, \frac{1}{r}+\frac{1}{s}=1$. If the above inequalities are reversed, then $h$ is said to be concave.

Definition 2.2. A function $h: I \rightarrow \mathbb{R}^{+}$is said to be log-convex on $I$ if

$$
(\ln h(x))^{\prime \prime} \geq 0,
$$

for all $x \in I$ or equivalently, if

$$
h\left(\frac{x}{r}+\frac{y}{s}\right) \leq[h(x)]^{\frac{1}{r}}[h(y)]^{\frac{1}{s}},
$$

for all $x, y \in I$, where $r>1, \frac{1}{r}+\frac{1}{s}=1$. If the above inequalities are reversed, then $h$ is said to be log-concave.

Definition 2.3 ( $[18])$. A function $h: I \rightarrow \mathbb{R}$ is said to be completely monotonic on $I$ if $h$ has derivatives of all orders on I and

$$
(-1)^{n} h^{(n)}(x) \geq 0,
$$

on I for all $x \in I$ and $n \in N$. If the inequality is strict, then $h$ is said to be strictly completely monotonic.

Definition 2.4 ([8]). A function $h: I \rightarrow \mathbb{R}$ is said to be subadditive on $I$ if

$$
h(x+y) \leq h(x)+h(y),
$$

for all $x, y \in I$. If the inequality is reversed, then $h$ is said to be superadditive.

Lemma 2.1 ( $[16])$. If the function $\frac{h(x)}{x}$ is increasing (decreasing) on an interval $I$ then $h$ is superadditive (subadditive) on I. 
Definition 2.5 ( [8]). A function $h: I \rightarrow \mathbb{R}$ is said to be starshaped on $I$ if

$$
h(\alpha x) \leq \alpha h(x)
$$

for all $x \in I$ ans $\alpha \in[0,1]$.

Lemma 2.2 ( [8]). A function $h: I \rightarrow \mathbb{R}$ is starshaped if and only if either one of the following conditions is satisfied:

(a) $h(x) / x$ is increasing on $I$,

(b) $h^{\prime}(x) \geq h(x) / x$ for all $x \in I$.

\section{Results And Discussions}

Definition 3.1. Let $\Psi(x)$ represent the logarithmic derivative of the Barnes G-function. That is

$$
\Psi(x)=\frac{d}{d x} \ln G(x)=\frac{G^{\prime}(x)}{G(x)}
$$

for all $x>0$.

Theorem 3.1. The function $\Psi(x)$ satisfies the identity

$$
\Psi(x)=\frac{1}{2} \ln (2 \pi)+\frac{1}{2}-x+(x-1) \psi(x),
$$

and the functional equation

$$
\Psi(x+1)=\Psi(x)+\psi(x)
$$

for $x>0$, where $\psi(x)$ is the classical digamma function.

Proof. By substituting (1.10) into (1.11), we obtain

$$
G(x)=\frac{1}{\Gamma(x)}\left\{(2 \pi)^{\frac{x}{2}} \exp \left(-\frac{x+x^{2}(1+\gamma)}{2}\right) \prod_{k=1}^{\infty}\left(1+\frac{x}{k}\right)^{k} \exp \left(\frac{x^{2}}{2 k}-x\right)\right\}
$$

and by taking logarithm of both sides, we obtain

$$
\ln G(x)=-\ln \Gamma(x)+\frac{x}{2} \ln (2 \pi)-\frac{1}{2}\left(x+x^{2}(1+\gamma)\right)+\sum_{k=1}^{\infty}\left(k \ln \left(1+\frac{x}{k}\right)+\frac{x^{2}}{2 k}-x\right)
$$


Next, by differentiating and using (1.5) we obtain

$$
\begin{aligned}
\Psi(x) & =\frac{1}{2} \ln (2 \pi)-\frac{1}{2}-x-\gamma x+x \sum_{k=1}^{\infty}\left(k \cdot \frac{1}{1+\frac{x}{k}} \cdot \frac{1}{k}+\frac{1}{k}-1\right)-\frac{\Gamma^{\prime}(x)}{\Gamma(x)} \\
& =\frac{1}{2} \ln (2 \pi)-\frac{1}{2}-x-\gamma x+\sum_{k=1}^{\infty}\left(\frac{k}{k+x}-1+\frac{x}{k}\right)-\psi(x) \\
& =\frac{1}{2} \ln (2 \pi)-\frac{1}{2}-x-\gamma x+x \sum_{k=1}^{\infty}\left(\frac{1}{k}-\frac{1}{k+x}\right)-\psi(x) \\
& =\frac{1}{2} \ln (2 \pi)-\frac{1}{2}-x+x \psi(x+1)-\psi(x) \\
& =\frac{1}{2} \ln (2 \pi)+\frac{1}{2}-x+(x-1) \psi(x),
\end{aligned}
$$

which gives (3.1). Finally, by taking the logarithmic derivative of (1.10), we obtain (3.2) and this completes the proof.

A plot of the function $\Psi(x)$ for $x>0$ is shown in Figure 1 below.

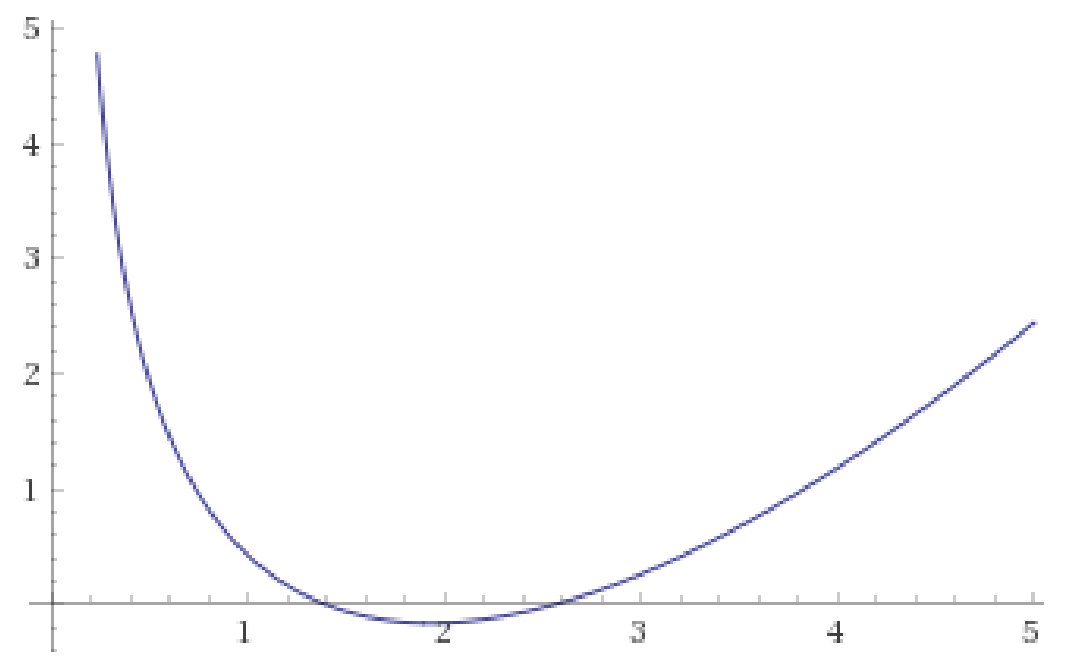

FIGURE 1. A plot of $\Psi(x)$.

Remark 3.1. The function $\Psi(x)$ has a minimum value of approximately -0.1608131817 which occurs at $x \approx 1.9258631233$. The only positive roots of $\Psi(x)$ are $x \approx 1.3914703810$ and $x \approx 2.5576639327$.

Remark 3.2. Some particular values of $\Psi(x)$ are derived as follows.

$$
\begin{gathered}
\Psi(1 / 2)=\frac{1}{2}(\gamma+\ln (8 \pi))=1.9006935462 \ldots \\
\Psi(1)=\frac{1}{2}(\ln (2 \pi)-1)=0.4189385332 \ldots
\end{gathered}
$$




$$
\begin{gathered}
\Psi(3 / 2)=\frac{1}{2}\left(\ln \left(\frac{\pi}{2}\right)-\gamma\right)=-0.0628164798 \ldots, \\
\Psi(2)=\frac{1}{2}(\ln (2 \pi)-1-2 \gamma)=-0.15827713169 \ldots, \\
\Psi(5 / 2)=\frac{1}{2}\left(4-3 \gamma+\ln \left(\frac{\pi}{32}\right)\right)=-0.0263265058 \ldots, \\
\Psi(3)=\frac{1}{2}(1-4 \gamma+\ln (2 \pi))=0.2645072034 \ldots, \\
\Psi(7 / 2)=-3+\frac{5}{2}\left(\frac{46}{15}-\gamma-\ln 4\right)+\frac{1}{2} \ln (2 \pi)=0.6768301348 \ldots, \\
\Psi(4)=-\frac{7}{2}+3\left(\frac{11}{6}-\gamma\right)+\frac{1}{2} \ln (2 \pi)=1.1872915385 \ldots
\end{gathered}
$$

Theorem 3.2. The function $\Psi(x)$ is decreasing on $\left(0, x_{0}\right)$ and is increasing on $\left(x_{0}, \infty\right)$, where $x_{0}=$ $1.4616321449 \ldots$ is the only positive root of the digamma function, $\psi(x)$.

Proof. Let $x \in(0, \infty)$ and $x_{0}=1.4616321449 \ldots$ be the only positive root of the digamma function $\psi(x)$. Then by (3.2), we have

$$
\Psi(x+1)-\Psi(x)=\psi(x)
$$

Since $\psi(x)<0$ for $0<x<x_{0}$ and $\psi(x)>0$ for $x>x_{0}$, we conclude that

$$
\Psi(x+1)-\Psi(x)<0,
$$

if $x \in\left(0, x_{0}\right)$ and

$$
\Psi(x+1)-\Psi(x)>0
$$

if $x \in\left(x_{0}, \infty\right)$. This concludes the proof.

Proposition 3.1. The $n$-th derivative of the function $\Psi(x)$ is given as

$$
\Psi^{(n)}(x)=x \psi^{(n)}(x)+n \psi^{(n-1)}(x)-\psi^{(n)}(x),
$$

for $x>0$ and $n \in \mathbb{N}$ where $n \geq 2$.

Proof. By differentiating (3.1), we obtain

$$
\begin{aligned}
\Psi^{\prime}(x) & =-1+x \psi^{\prime}(x)+\psi(x)-\psi^{\prime}(x), \\
\Psi^{\prime \prime}(x) & =x \psi^{\prime \prime}(x)+2 \psi^{\prime}(x)-\psi^{\prime \prime}(x), \\
\Psi^{\prime \prime \prime}(x) & =x \psi^{\prime \prime \prime}(x)+3 \psi^{\prime \prime}(x)-\psi^{\prime \prime \prime}(x) .
\end{aligned}
$$


Continuing the process for $n$ number of times yields

$$
\Psi^{(n)}(x)=x \psi^{(n)}(x)+n \psi^{(n-1)}(x)-\psi^{(n)}(x),
$$

which completes the proof.

Proposition 3.2. The function $\Psi(x)$ satisfies the identities

$$
\begin{aligned}
\Psi(x) & =\frac{1}{2} \ln (2 \pi)+\frac{1}{2}-x-(x-1) \gamma+(x-1) \sum_{k=0}^{\infty}\left(\frac{1}{k+1}-\frac{1}{k+x}\right), \\
& =\frac{1}{2} \ln (2 \pi)+\frac{1}{2}-x+(x-1) \int_{0}^{\infty}\left(\frac{e^{-t}}{t}-\frac{e^{-x t}}{1-e^{-t}}\right) d t
\end{aligned}
$$

for $x>0$.

Proof. This follows directly by using (3.1) in conjunction with (1.2) and (1.4) respectively.

Theorem 3.3. The function $\Psi^{\prime \prime}(x)$ is completely monotonic on $(0, \infty)$.

Proof. By using (1.8) and (3.3), we obtain

$$
\begin{aligned}
\Psi^{(n+2)}(x) & \\
= & x(-1)^{n+3}(n+2) ! \sum_{k=0}^{\infty} \frac{1}{(k+x)^{n+3}}+(n+2)(-1)^{n+2}(n+1) ! \sum_{k=0}^{\infty} \frac{1}{(k+x)^{n+2}} \\
& -(-1)^{n+3}(n+2) ! \sum_{k=0}^{\infty} \frac{1}{(k+x)^{n+3}} \\
= & (-1)^{n}(n+2) ! \sum_{k=0}^{\infty}\left[\frac{-x}{(k+x)^{n+3}}+\frac{1}{(k+x)^{n+2}}+\frac{1}{(k+x)^{n+3}}\right] \\
= & (-1)^{n}(n+2) ! \sum_{k=0}^{\infty} \frac{k+1}{(k+x)^{n+3}} .
\end{aligned}
$$

Now, multiplying through by $(-1)^{n}$ gives

$$
(-1)^{n} \Psi^{(n+2)}(x)=(n+2) ! \sum_{k=0}^{\infty} \frac{k+1}{(k+x)^{n+3}}>0,
$$

which completes the proof.

Theorem 3.4. The function $\Psi(x)$ is strictly convex for all $x \in(0, \infty)$. 
Proof. By using (3.3) together with (1.2), we obtain

$$
\begin{aligned}
\Psi^{\prime \prime}(x) & =x \psi^{\prime \prime}(x)+2 \psi^{\prime}(x)-\psi^{\prime \prime}(x) \\
& =-x \sum_{k=0}^{\infty} \frac{2}{(k+x)^{3}}+2 \sum_{k=0}^{\infty} \frac{1}{(k+x)^{2}}+\sum_{k=0}^{\infty} \frac{2}{(k+x)^{3}} \\
& =2 \sum_{k=0}^{\infty} \frac{k+1}{(k+x)^{3}} \\
& >0
\end{aligned}
$$

which completes the proof.

Lemma 3.1. Let $h(x)=x-\frac{1}{2} \ln (2 \pi)-\frac{1}{2}+\psi(x)$ and $c \approx 1.44004 \ldots$ be the only positive root of $h(x)$. Then, $h(x)<0$ if $x \in(0, c)$ and $h(x)>0$ if $x \in(c, \infty)$.

Proof. Let $x \in(0, \infty)$ and $h(x)=x-\frac{1}{2} \ln (2 \pi)-\frac{1}{2}+\psi(x)$. Then

$$
h^{\prime}(x)=1+\psi^{\prime}(x)>0
$$

which implies that $h(x)$ is increasing. Then for $x \in(0, c)$, we have $h(x)<h(c)=0$ and for $x \in(c, \infty)$ implies we have $h(x)>h(c)=0$. This completes the proof.

Theorem 3.5. Let $c$ be as defined in Lemma 3.1. Then the function $\frac{\Psi(x)}{x}$ is decreasing on $(0, c)$ and increasing on $(c, \infty)$.

Proof. By (3.1), we have

$$
\begin{aligned}
& \frac{\Psi(x+1)}{x+1}-\frac{\Psi(x)}{x} \\
& =\frac{\frac{1}{2} \ln (2 \pi)+\frac{1}{2}-x+x \psi(x)}{x+1}-\frac{\frac{1}{2} \ln (2 \pi)+\frac{1}{2}-x+x \psi(x)-\psi(x)}{x} \\
& =\frac{x\left[\frac{1}{2} \ln (2 \pi)+\frac{1}{2}-x+x \psi(x)\right]-(x+1)\left[\frac{1}{2} \ln (2 \pi)+\frac{1}{2}-x+x \psi(x)-\psi(x)\right]}{x(x+1)} \\
& =\frac{x-\frac{1}{2} \ln (2 \pi)-\frac{1}{2}+\psi(x)}{x(x+1)}=Q(x) .
\end{aligned}
$$

By applying Lemma 3.1, $Q(x)<0$ if $x \in(0, c)$ and $Q(x)>0$ if $x \in(c, \infty)$. This completes the proof.

Corollary 3.1. Let $c$ be as defined in Lemma 3.1. Then $\Psi(x)$ is starshaped on $(c, \infty)$. That is

$$
\Psi(\lambda x) \leq \lambda \Psi(x)
$$

where $\lambda \in[0,1]$ and $x \in(c, \infty)$. 
Proof. This follows from Lemma 2.2 and Theorem 3.5.

Corollary 3.2. Let $c$ be as defined in Lemma 3.1. Then $\Psi(x)$ is subadditive on $(0, c)$ and superadditive on $(c, \infty)$. In other words,

$$
\Psi(x+y) \leq \Psi(x)+\Psi(y)
$$

if $x, y \in(0, c)$ and

$$
\Psi(x+y) \geq \Psi(x)+\Psi(y)
$$

if $x, y \in(c, \infty)$.

Proof. This follows from Lemma 2.1 and Theorem 3.5.

Theorem 3.6. The function $\Psi^{\prime \prime}(x)$ is strictly decreasing on $(0, \infty)$.

Proof. By using (3.3) and (1.2), we obtain

$$
\begin{aligned}
\Psi^{\prime \prime \prime}(x) & =x \psi^{\prime \prime \prime}(x)+3 \psi^{\prime \prime}(x)-\psi^{\prime \prime \prime}(x) \\
& =6 x \sum_{k=0}^{\infty} \frac{1}{(k+x)^{4}}-6 \sum_{k=0}^{\infty} \frac{1}{(k+x)^{3}}-6 \sum_{k=0}^{\infty} \frac{1}{(k+x)^{4}} \\
& =-6 \sum_{k=0}^{\infty} \frac{k+1}{(k+x)^{4}}<0,
\end{aligned}
$$

which completes the proof.

Theorem 3.7. The function $\Psi^{\prime \prime}(x)$ is subadditive on $(0, \infty)$.

Proof. By using (3.3) and (1.2), we obtain

$$
\begin{aligned}
\left\{\frac{\Psi^{\prime \prime}(x)}{x}\right\}^{\prime} & =\frac{x \Psi^{\prime \prime \prime}(x)-\Psi^{\prime \prime}(x)}{x^{2}} \\
& =-\frac{1}{x^{2}}\left[6 x \sum_{k=0}^{\infty} \frac{k+1}{(k+x)^{4}}+2 \sum_{k=0}^{\infty} \frac{k+1}{(k+x)^{3}}\right] \\
& <0 .
\end{aligned}
$$

Hence $\frac{\Psi^{\prime \prime}(x)}{x}$ is decreasing on $(0, \infty)$. Then by Lemma 2.1, we conclude that, $\Psi^{\prime \prime}(x)$ is subadditive on $(0, \infty)$

Theorem 3.8. For $a>1$, the function $\alpha(x)=\frac{G(a x)}{[G(x)]^{a}}$ is decreasing on $\left(0, x_{0}\right)$ and increasing on $\left(x_{0}, \infty\right)$, where $x_{0}=1.4616321449 \ldots$ is the only positive root of the digamma function, $\psi(x)$. 
Proof. Let $a>1$ and $x \in(0, \infty)$. Then logarithmic derivative of $\alpha(x)$ yields

$$
\frac{\alpha^{\prime}(x)}{\alpha(x)}=\left[\ln \frac{G(a x)}{[G(x)]^{a}}\right]^{\prime}=a\left[\frac{G^{\prime}(a x)}{G(a x)}-\frac{G^{\prime}(x)}{G(x)}\right] .
$$

We know from Theorem 3.2 that $\Psi(x)=\frac{G^{\prime}(x)}{G(x)}$ is decreasing on $\left(0, x_{0}\right)$ and increasing on $\left(x_{0}, \infty\right)$. Hence

if $x \in\left(0, x_{0}\right)$ and

$$
\frac{G^{\prime}(a x)}{G(a x)}-\frac{G^{\prime}(x)}{G(x)}<0
$$

$$
\frac{G^{\prime}(a x)}{G(a x)}-\frac{G^{\prime}(x)}{G(x)}>0
$$

if $x \in\left(x_{0}, \infty\right)$. Thus, $\alpha^{\prime}(x)<0$ if $x \in\left(0, x_{0}\right)$ and $\alpha^{\prime}(x)>0$ if $x \in\left(x_{0}, \infty\right)$. This completes the proof.

Remark 3.3. If $0<a<1$, then $\alpha(x)$ is increasing on $\left(0, x_{0}\right)$ and decreasing on $\left(x_{0}, \infty\right)$.

Corollary 3.3. For $a>1$ and $0<x<y<x_{0}$, the inequality

$$
\left[\frac{G(y)}{G(x)}\right]^{a}>\frac{G(a y)}{G(a x)}
$$

is satisfied.

Proof. Let $a>1$ and $0<x<y<x_{0}$. Then, since $\alpha(x)=\frac{G(a x)}{[G(x)]^{a}}$ is decreasing on $\left(0, x_{0}\right)$, we have

which when rearranged gives (3.9).

$$
\frac{G(a x)}{[G(x)]^{a}}>\frac{G(a y)}{[G(y)]^{a}},
$$

Remark 3.4. Inequality (3.9) is reversed if $x_{0}<x<y<\infty$.

Theorem 3.9. Let $a>1$. Then the function $\beta(x)=\frac{[G(x+1)]^{a}}{G(a x+1)}$ is increasing on $\left(0, x_{0}\right)$ and decreasing on $\left(x_{0}, \infty\right)$.

Proof. Let $a>1$ and $x \in(0, \infty)$. Then logarithmic derivative of $\beta(x)$ yields

$$
\frac{\beta^{\prime}(x)}{\beta(x)}=a\left[\frac{G^{\prime}(x+1)}{G(x+1)}-\frac{G^{\prime}(a x+1)}{G(a x+1)}\right] .
$$

Since $\frac{G^{\prime}(x)}{G(x)}$ is decreasing on $\left(0, x_{0}\right)$ and increasing on $\left(x_{0}, \infty\right)$, then

$$
\frac{G^{\prime}(x+1)}{G(x+1)}-\frac{G^{\prime}(a x+1)}{G(a x+1)}>0
$$

if $x \in\left(0, x_{0}\right)$ and

$$
\frac{G^{\prime}(x+1)}{G(x+1)}-\frac{G^{\prime}(a x+1)}{G(a x+1)}<0
$$


if $x \in\left(x_{0}, \infty\right)$. Thus, $\beta^{\prime}(x)>0$ if $x \in\left(0, x_{0}\right)$ and $\beta^{\prime}(x)<0$ if $x \in\left(x_{0}, \infty\right)$. This completes the proof.

Corollary 3.4. For $a>1$ and $x \in(0,1)$, the inequality

$$
1<\frac{[G(x+1)]^{a}}{G(a x+1)}<\frac{1}{G(a+1)},
$$

is satisfied.

Proof. Since $\beta(x)=\frac{[G(x+1)]^{a}}{G(a x+1)}$ is increasing on $\left(0, x_{0}\right)$, then for $x \in(0,1)$, we have

$$
\beta(0)<\beta(x)<\beta(1)
$$

which yields (3.10).

Remark 3.5. Results similar to Corollary 3.4, for the gamma function, can be found in Corollary 2.4 of $[17]$.

Corollary 3.5. The Barnes $G$-function $G(x)$ is log-concave on $\left(0, x_{0}\right)$ and log-convex on $\left(x_{0}, \infty\right)$.

Proof. It is easily realized that $(\ln G(x))^{\prime \prime}=\Psi^{\prime}(x)$. Then the conclusion is drawn from Theorem 3.2 .

Remark 3.6. Corollary 3.5 is an inprovement of Theorem 1 of [13].

Remark 3.7. Corollary 3.5 implies that

$$
G^{\prime \prime}(x) G(x) \geq\left(G^{\prime}(x)\right)^{2}
$$

for $x \in\left(x_{0}, \infty\right)$ and

$$
G\left(\frac{x}{r}+\frac{y}{s}\right) \leq[G(x)]^{\frac{1}{r}}[G(y)]^{\frac{1}{s}},
$$

for $x, y \in\left(x_{0}, \infty\right), r>1$ and $\frac{1}{r}+\frac{1}{s}=1$. For $x, y \in\left(0, x_{0}\right)$, inequalities (3.11) and (3.12) are reversed.

\section{Conclusions}

In this paper, we have established an explicit representation for the logarithmic derivative of the Barnes $G$-function which we denoted by $\Psi(x)$. The representation is in terms of the classical digamma function. We further established some analytical properties such as monotonicity, complete monotonicity, convexity, log-convexity, subadditivity, superadditivity and starshapedness of the function $\Psi(x)$. Finally, we deduced some inequalities involving 
the Barnes $G$-function. It is expected that, this work will lay a good foundation for further investigations on the the Barnes $G$-function and its logarithmic derivative.

\section{REFERENCES}

[1] M. Abramowitz and I. A. Stegun, Handbook of Mathematical Functions With Formulas, Graphs, and Mathematical Tables, National Bureau of Standards Applied Mathematics Series 55, John Wiley and Sons, New York, 10th edition, 1972.

[2] V. S. Adamchik, Contributions to the Theory of the Barnes Function, Int. J. Math. Comput. Sci., 9(1)(2014), 11-30.

[3] E. Artin, The Gamma Function, Holt, Rinehart and Winston, Inc., USA. 1964.

[4] E. W. Barnes, The theory of G-function, Quart. J. Math. 31 (1899), 264-314.

[5] E. W. Barnes, On the theory of the multiple gamma function, Trans. Cambridge Philos. Soc., 19(1904), 374-425.

[6] N. Batir, Inequalities for the double gamma function, J. Math. Anal. Appl., 351(1)(2009), 182-185.

[7] N. Batir and M. Cancan, A Double Inequality for the Double Gamma Function, Int. J. Math. Anal., 2(7) (2008), 329-335.

[8] A. M. Bruckner and E. Ostrow, Some function classes related to the class of convex functions, Pac. J. Math., $12(4)(1962), 1203-1215$.

[9] C-P. Chen, Inequalities associated with Barnes G-function, Expo. Math., 29(2011),119-125.

[10] J. Choi, A Duplication Formula for the Double Gamma Function $\Gamma_{2}$, Bull. Korean Math. Soc., 33(2) (1996), 289-294.

[11] J. Choi and H. M. Srivastava, A Note on Multiplication Formular for the Multiple Gamma Function $\Gamma_{n}$, Ital. J. Pure Appl. Math., 23(2008), 179-188.

[12] D. F. Connon, An introduction to the Barnes double gamma function with an application to an integral involving the cotangent function, arXiv:1801.08025v3 [math.CA].

[13] S. Das and A. Swaminathan, Bounds for triple gamma functions and their ratios, J. Inequal. Appl., 2016 (2016), 210.

[14] C. Ferreira and J. Lopez, An Asymptotic Expansion of the Double Gamma Function, J. Approx. Theory, $111(2)(2001), 298-314$.

[15] I.S. Gradshteyn and I.M. Ryzhik, Table of Integrals, Series, and Products (7th Edition), Academic Press, 2007.

[16] B. Ravi and A. V. Laxmi, Subadditive and completely monotonic properties of the tricomi confluent hypergeometric functions, Int. J. Adv. Math. 2018(5)(2018), 25-33.

[17] J. Sandor, A note on certain inequalities for the gamma function, Ineq. Pure Appl. Math., 6(3)(2005), 61.

[18] D. V. Widder, The Laplace Transform, Princeton University Press, London, 1941.

[19] Z. Xu and W. Wang, More asymptotic expansions for the Barnes G-function, J. Number Theory, 174(2017), 505-517. 